\title{
LncRNAs and their regulatory networks in breast muscle tissue of Chinese Gushi chickens during late postnatal development
}

\author{
Yuanfang $\mathrm{Li}^{1 \dagger}$, Wenjiao $\mathrm{Jin}^{1+}$, Bin Zhai ${ }^{1}$, Yi Chen ${ }^{1}$, Guoxi Li ${ }^{1,2^{*}}$, Yanhua Zhang ${ }^{1}$, Yujie Guo ${ }^{1}$, Guirong Sun ${ }^{1,2}$,
} Ruili Han ${ }^{1,2}$, Zhuanjian $\mathrm{Li}^{1,2}$, Hong Li ${ }^{1,2}$, Yadong Tian ${ }^{1,2}$, Xiaojun Liu ${ }^{1,2}$ and Xiangtao Kang ${ }^{1,2^{*}}$

\begin{abstract}
Background: Chicken skeletal muscle is an important economic product. The late stages of chicken development constitute the main period that affects meat production. LncRNAs play important roles in controlling the epigenetic process of growth and development. However, studies on the role of IncRNAs in the late stages of chicken breast muscle development are still lacking. In this study, to investigate the expression characteristics of IncRNAs during chicken muscle development, 12 cDNA libraries were constructed from Gushi chicken breast muscle samples from 6-, 14-, 22-, and 30-week-old chickens.

Results: A total of 1252 new IncRNAs and 1376 annotated IncRNAs were identified. Furthermore, 53, 61, 50, 153, 117 , and 78 DE-IncRNAs were found in the W14 vs. W6, W22 vs. W14, W22 vs. W6, W30 vs. W6, W30 vs. W14, and W30 vs. W22 comparison groups, respectively. After GO enrichment analysis of the DE-IncRNAs, several muscle development-related GO terms were found in the W22 vs. W14 comparison group. Moreover, it was found that the MAPK signaling pathway was one of the most frequently enriched pathways in the different comparison groups. In addition, 12 common target DE-miRNAs of DE-IncRNAs were found in different comparison groups, some of which were muscle-specific miRNAs, such as gga-miR-206, gga-miR-1a-3p, and miR-133a-3p. Interestingly, the precursors of four newly identified miRNAs were found to be homologous to IncRNAs. Additionally, we found some ceRNA networks associated with muscle development-related GO terms. For example, the ceRNA networks contained the DYNLL2 gene with 12 IncRNAs that targeted 2 miRNAs. We also constructed PPI networks, such as IGF-I-EGF and FZD6-WNT11.

Conclusions: This study revealed, for the first time, the dynamic changes in IncRNA expression in Gushi chicken breast muscle at different periods and revealed that the MAPK signaling pathway plays a vital role in muscle development. Furthermore, MEF2C and its target IncRNA may be involved in muscle regulation through the MAPK signaling pathway. This research provided valuable resources for elucidating posttranscriptional regulatory mechanisms to promote the development of chicken breast muscles after hatching.
\end{abstract}

Keywords: Chicken, Breast muscle, IncRNAs, Regulatory network, ceRNA

\footnotetext{
* Correspondence: liguoxi0914@126.com; xtkang2001@263.net

${ }^{\dagger}$ Yuanfang Li and Wenjiao Jin contributed equally to this work.

'College of Animal Science and Veterinary Medicine, Henan Agricultural University, Zhengzhou 450002, China

Full list of author information is available at the end of the article
}

C C The Author(s). 2021 Open Access This article is licensed under a Creative Commons Attribution 4.0 International License, which permits use, sharing, adaptation, distribution and reproduction in any medium or format, as long as you give appropriate credit to the original author(s) and the source, provide a link to the Creative Commons licence, and indicate if changes were made. The images or other third party material in this article are included in the article's Creative Commons licence, unless indicated otherwise in a credit line to the material. If material is not included in the article's Creative Commons licence and your intended use is not permitted by statutory regulation or exceeds the permitted use, you will need to obtain permission directly from the copyright holder. To view a copy of this licence, visit http://creativecommons.org/licenses/by/4.0/ The Creative Commons Public Domain Dedication waiver (http://creativecommons.org/publicdomain/zero/1.0/) applies to the data made available in this article, unless otherwise stated in a credit line to the data. 


\section{Background}

Muscle, especially skeletal muscle, is an important part of an animal [1]. In livestock production, skeletal muscle is an important economic product for human consumption. After birth, muscle weight continuously increases, and the growth of skeletal muscle is mainly achieved by increasing the hypertrophy of existing muscle fibers [2]. Compared with the embryonic stage, from hatching to marketing or elimination (referred to as late postnatal development), the development of chickens at this stage is an important period affecting meat production [3]; therefore, it is essential to study skeletal muscle growth and development during the postnatal late development of important agricultural species.

Muscle development is a complex multistage process in which many genes cooperate to regulate each stage [4]. Several candidate genes, such as the growth hormone secretagogue receptor (GHSR) [5], insulin-like growth factors (IGFs) [6], transforming growth factor beta 2 (TGF 2 ) [7], and myocyte enhancer factor $2 \mathrm{~B}(M E F 2 B)$ [8], have been identified to play important roles in the growth of chickens. Although many genes play important roles in chicken muscle growth, studies have shown that only a small percentage $(1-2 \%)$ of the genome encodes proteins in mammals, and tens of thousands of intergenic sites are transcribed into noncoding RNA [9]. In the past few years, regulatory RNAs, such as miRNAs, piRNAs, snoRNAs, and long noncoding RNAs (IncRNAs), have appeared to play roles in many important biological processes [10]. In complex organisms, lncRNAs contain hidden regulatory information that can play a role in the regulation of gene expression [11]; therefore, lncRNAs are important molecules that can affect the growth and development of chicken skeletal muscle.

LncRNAs are a class of non-protein-coding transcripts that are more than $200 \mathrm{bp}$ in length [12]. Several lncRNAs have been shown to be expressed during development and have been shown to play an important role in epigenetic processes that control differentiation and development. For example, as one of the earliest identified lncRNAs, H19 also plays a regulatory role in various growth and development processes [13]. MUNC is a IncRNA that promotes skeletal muscle production by stimulating the adjacent myogenic differentiation antigen $(M y o D)$ gene in C2C12 cells [14]. A novel lncRNA, Irm, has been shown to interact with $M E F 2 D$ to enhance myogenic differentiation [15]. LncRNAs can exert cisregulatory effects in biological processes. For instance, it has been indicated that IncRNA-Six 1 cis regulates the Six 1 gene and encodes a micropeptide to activate Six1, thereby promoting cell proliferation and participating in muscle growth [16]. In addition, IncRNAs can also play a trans-regulatory role in biological processes. For example, the noncoding RNA H19 can be used as a trans- regulator of $I G F 2$ [17]. Moreover, lncRNAs can also act as ceRNAs to protect mRNAs and act as a molecular sponge to inhibit miRNA targeting of mRNAs. For example, IncIRS1 acts as a sponge of the miR-15 family, regulating the expression of insulin receptor substrate 1 (IRS1), thereby promoting skeletal muscle production [18]. Although an increasing number of lncRNAs have been characterized by high-throughput sequencing studies, there are few reports on the regulation of lncRNAs in chicken muscle development. Therefore, it is important to study the expression characteristics of lncRNAs during chicken muscle development.

Gushi chicken is an excellent variety of egg- and meatproviding chicken native to Gushi County, Henan Province, China. Gushi chicken is tender and delicious, with a fresh and unique flavor, and eliminated hens are often used as meat. Although Gushi chickens have many excellent characteristics, their growth rate is slightly slower than that of commercial broilers. Our previous histological study of this type of breast muscle showed that before 22 weeks of age, muscle fiber diameter grew rapidly, and after 22 weeks of age, the relationship between the diameter and density of the breast muscle fibers remained balanced [19]. To understand and control the growth and development of Gushi chicken skeletal muscle, we must understand the molecular regulation mechanism of different stages of skeletal muscle development. In this study, we identified lncRNAs by deep-sequencing data from four different stages $(6,14,22$, and 30 weeks) of Gushi chicken skeletal muscle development. Differentially expressed lncRNAs were used to predict cis- and trans-target genes to construct potential lncRNA-mRNA interaction networks and explore important signaling pathways. Then, the lncRNA data were combined with mRNA and miRNA data to construct potential lncRNA-miRNA-mRNA interaction networks and explore the regulatory networks that play a role in chicken skeletal muscle development. In summary, this study identifies differentially expressed lncRNAs at different stages of postnatal late developmental and provides predictions about the associated interaction networks, which can be used to further study the molecular regulation mechanism of chicken muscle development.

\section{Results \\ Identification and characterization of IncRNAs}

Based on the Illumina HiSeq 2500 platform, a minimum of $89,496,872$ raw reads were obtained from each library, with a clean base ranging from $12.75 \mathrm{~Gb}$ to $16.66 \mathrm{~Gb}$ and an error rate of 0.01 or 0.02 (Table S1). To generate a complete annotation of the noncoding transcriptome of the Gushi chicken breast muscle tissue beyond the currently annotated transcriptome, we first used Cuffmerge [20] to combine and then screen the transcripts 
from each sample. In this study, a total of 20,438 transcripts were identified, 16,342 of which were mRNAs. In addition, a total of 1376 lncRNAs had been previously annotated, 1252 novel lncRNAs were identified (Fig. 1a, b), and 1468 transcripts of uncertain coding potential
(TUCPs) were screened. Only two types of lncRNAs were identified: an overwhelming majority of long intergenic noncoding RNAs (lincRNA) (79.7\%) and a minority of antisense lncRNAs (20.3\%) (Fig. 1c). LncRNAs in breast muscle tissue had a lower total transcript length,

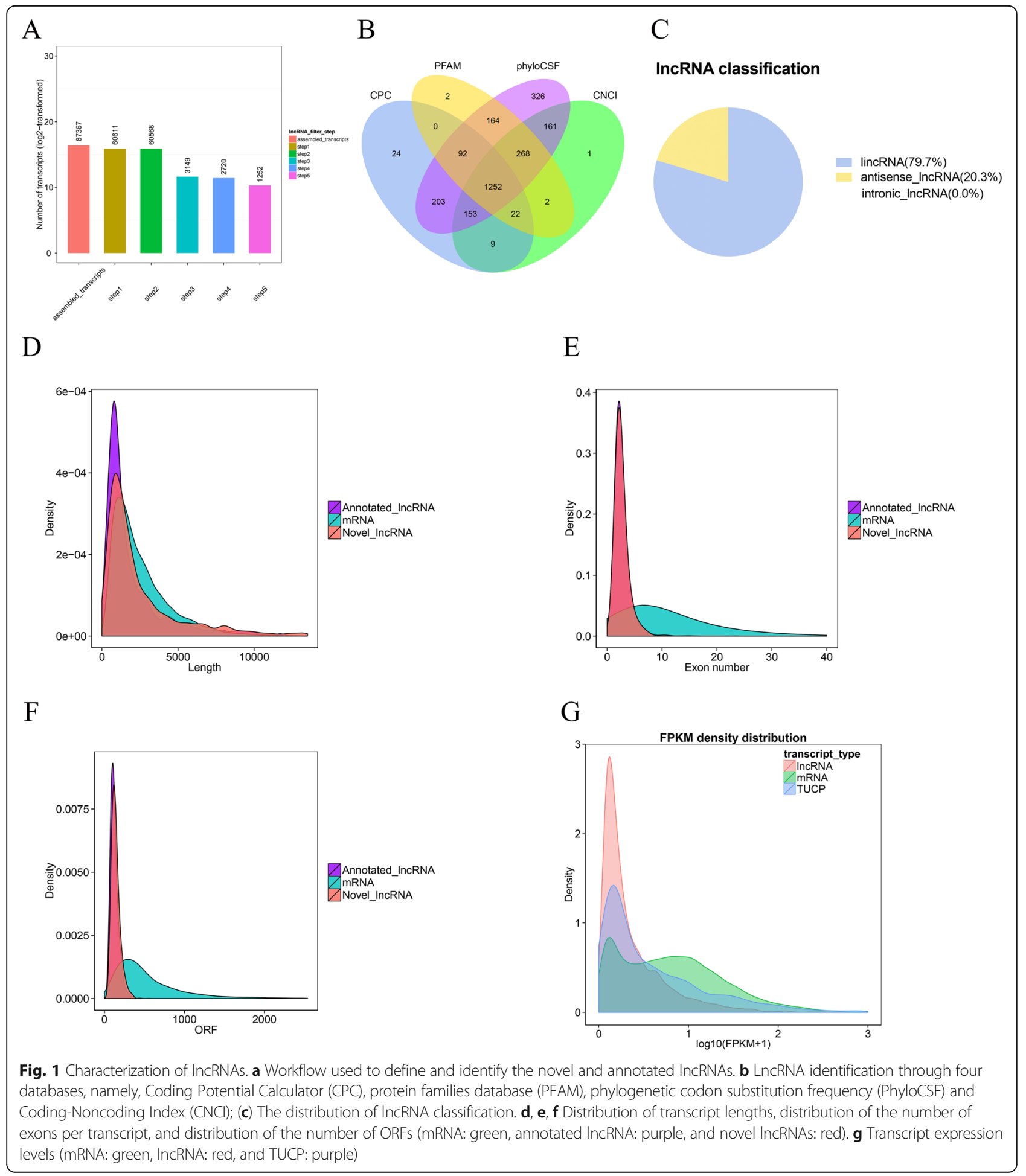


fewer exons, and fewer open reading frame (ORF) numbers than mRNAs (Fig. 1d-f) and a lower average transcript abundance (Fig. 1g).

\section{Characteristics of differentially expressed IncRNAs}

To gain insight into the key lncRNAs involved in chicken breast muscle development, we analyzed the differentially expressed lncRNAs (DE-lncRNAs) (|fold change, $\mathrm{FC} \mid \geq 1.7, q$-value $<0.05)$ at four different developmental stages, namely, 6 weeks (W6), 14 weeks (W14), 22 weeks (W22) and 30 weeks (W30). Among the six different comparison groups, there were 53, 61, 50, 153, 117 , and 78 DE-IncRNAs in the W14 vs. $W 6, W 22$ vs.
$W 14, W 22$ vs. $W 6, W 30$ vs. $W 6, W 30$ vs. $W 14$, and $W 30$ vs. W22 comparison groups, respectively. Venn diagram analysis showed that there were no common DElncRNAs among the six comparison groups (Fig. 2). Only LNC_000920 was commonly found in the following five comparison groups: $W 14$ vs. W6, W22 vs. W14, $W 30$ vs. W6, $W 30$ vs. $W 14$, and $W 30$ vs. W22. Moreover, LNC_000255 appeared in the following comparison groups: $W 14$ vs. $W 6, W 22$ vs. $W 14, W 22$ vs. $W 6, W 30$ vs. $W 6$, and $W 30$ vs. W14. Then, the DE-lncRNAs were identified by a DEGseq (differentially expressed gene, DEG) analysis, and DE-lncRNAs were clustered based on their expression profiles (Fig. S1). The clustering

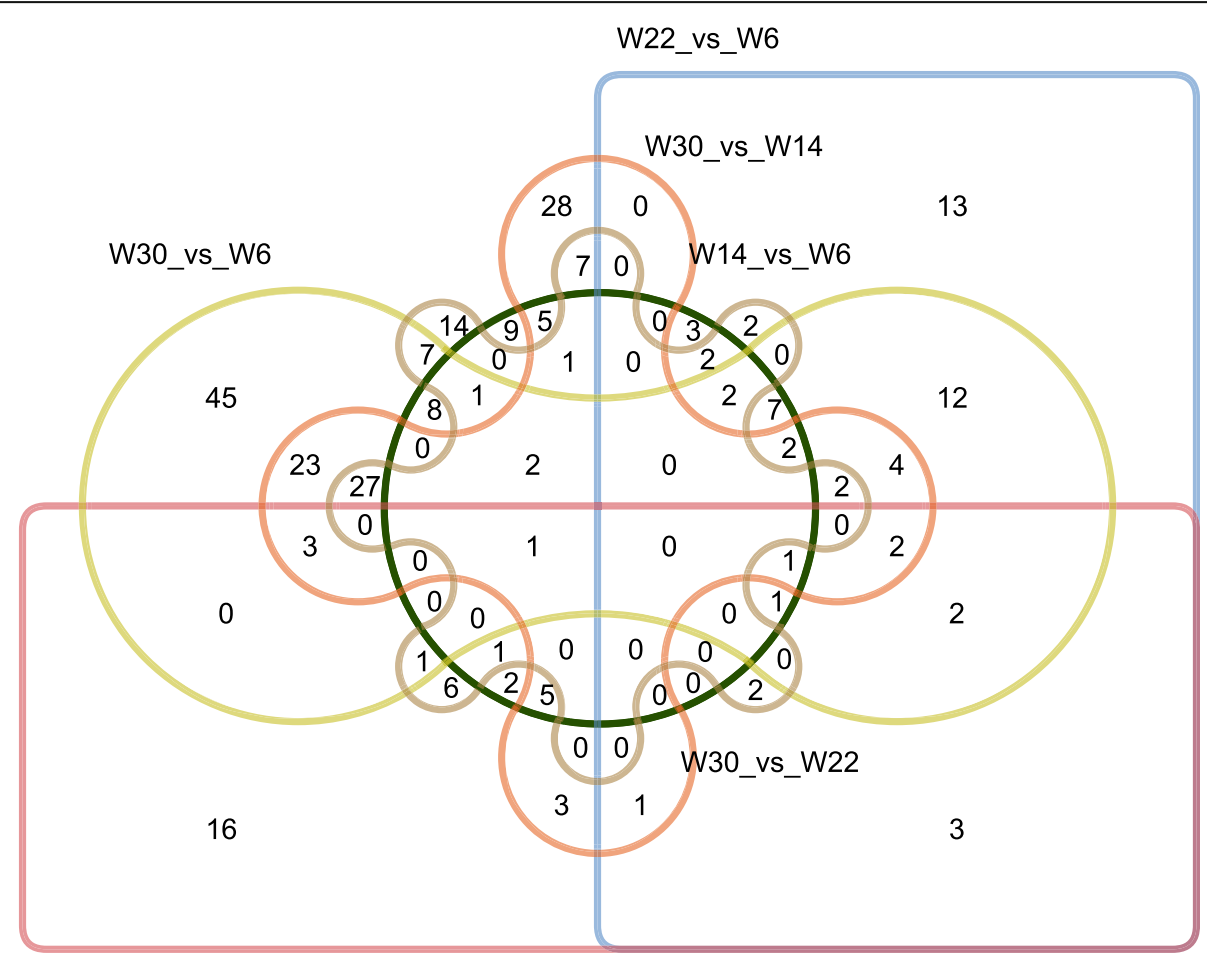

W22_vs_W14

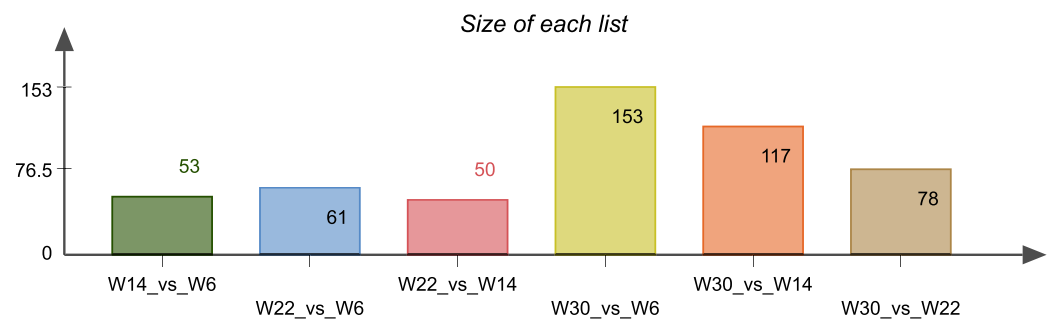

Number of elements: specific (1) or shared by 2, 3, ... lists

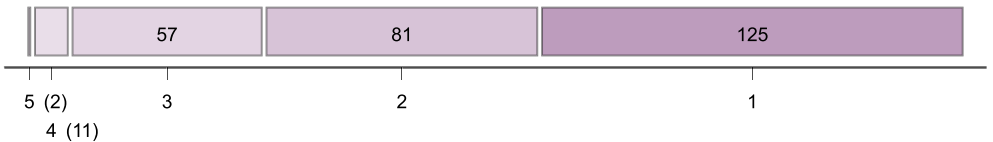

Fig. 2 Venn diagram of DE-IncRNAs in six comparison groups 
results showed that the intragroup repeats of each group were clustered together, indicating that the intragroup differences were smaller than the intergroup differences, which proved that the data were reliable. In addition, we found that 22 weeks clustered close to 14 weeks, followed by 6 weeks, and the farthest distance was from 30 weeks. In addition, we also found that the number of common DE-lncRNAs between W22 and W14 was the lowest (Fig. 2). It is possible that from 14 weeks - 22 weeks, many of the same lncRNAs played a common role, which also led to the reduction in DE-lncRNA that was commonly seen in the six comparison groups. Moreover, after identifying DE-lncRNAs, we analyzed the chromosome distribution information of the DE-lncRNAs and found that DE-lncRNAs were distributed in almost all chromosomes but not in chromosomes 22 and 27, and the largest number was found in chromosome 1 (Fig. S2). In addition, we selected several lncRNAs for data validation. The lncRNA expression level was determined and showed a similar pattern to that of the RNA-seq data (Fig. 3), indicating that the RNA-seq data were authentic.

To investigate the possible functions of the DElncRNAs in breast muscle between the different developmental stages, we conducted Gene Ontology (GO, http://www.geneontology.org/) enrichment analysis to uncover the enriched biological process terms associated with DE-lncRNA-targeted DEGs for each comparison group. Only the top $20 \mathrm{GO}$ terms for the $W 14$ vs. $W 6, W 22$ vs. $W 14$, and $W 30$ vs. $W 22$ comparison groups are shown in Fig. 4 and Fig. S3. The cis-targets of all lncRNAs were predicted with a 100$\mathrm{kb}$ upstream and downstream range. The GO enrichment analysis of the cis-targets of lncRNAs showed that only one growth- and developmentrelated GO term, called positive regulation of embryonic development, was found in the W22 vs. W14 comparison group (Fig. 4a). In addition, we predicted the regulation in trans between lncRNAs and genes by the Pearson correlation coefficient $r>0.95$. In the GO analysis of the trans-targets of lncRNAs, we found several muscle development-related GO terms only in the W22 vs. W14 comparison group (Fig. 4b), such as positive regulation of skeletal muscle tissue development, positive regulation of striated muscle tissue development, positive regulation of muscle organ development, positive regulation of muscle tissue development, and positive regulation of striated muscle cell differentiation.

To further understand how DE-lncRNA-targeted DEGs play roles in regulating chicken muscle development, we performed Kyoto Encyclopedia of Genes and Genomes (KEGG, http://www.genome.jp/kegg/) pathway analysis for each comparison. In the KEGG pathway analysis of the cis-targets of lncRNAs, the phagosome pathway was identified as the most significantly enriched pathway for the W14 vs. W6 comparison group (Fig. S4A). Furthermore, for the W22 vs. W14 comparison group, the endocytosis pathway was identified as the most significantly enriched pathway (Fig. S4B). Additionally, the focal adhesion and cytokine-cytokine receptor interaction pathways were the top two pathways for the W30 vs. W22 comparison group (Fig. S4C). Moreover, the KEGG pathway analysis of the trans-targets of IncRNAs showed that the propanoate metabolism pathway and fatty acid metabolism pathway were the top two pathways for

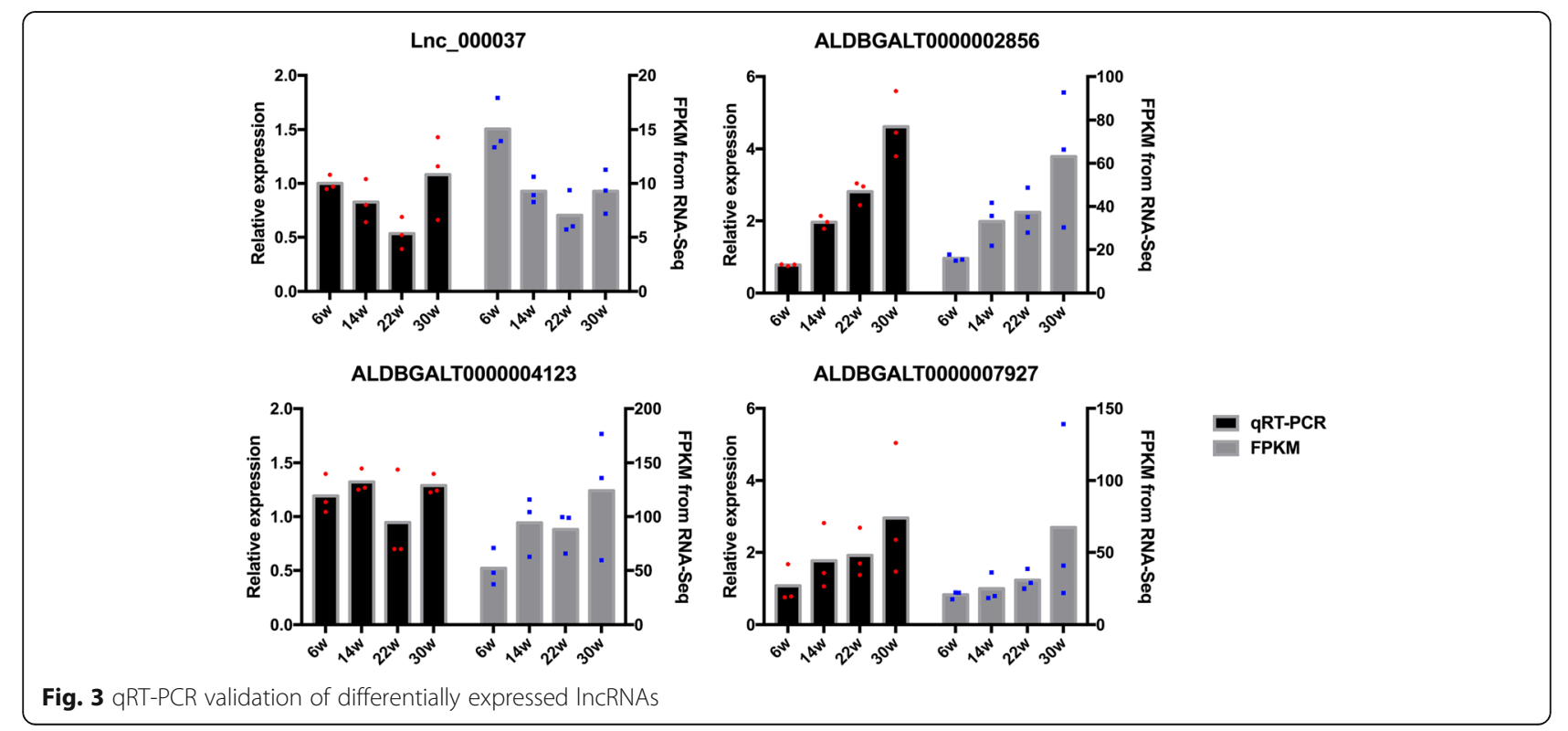



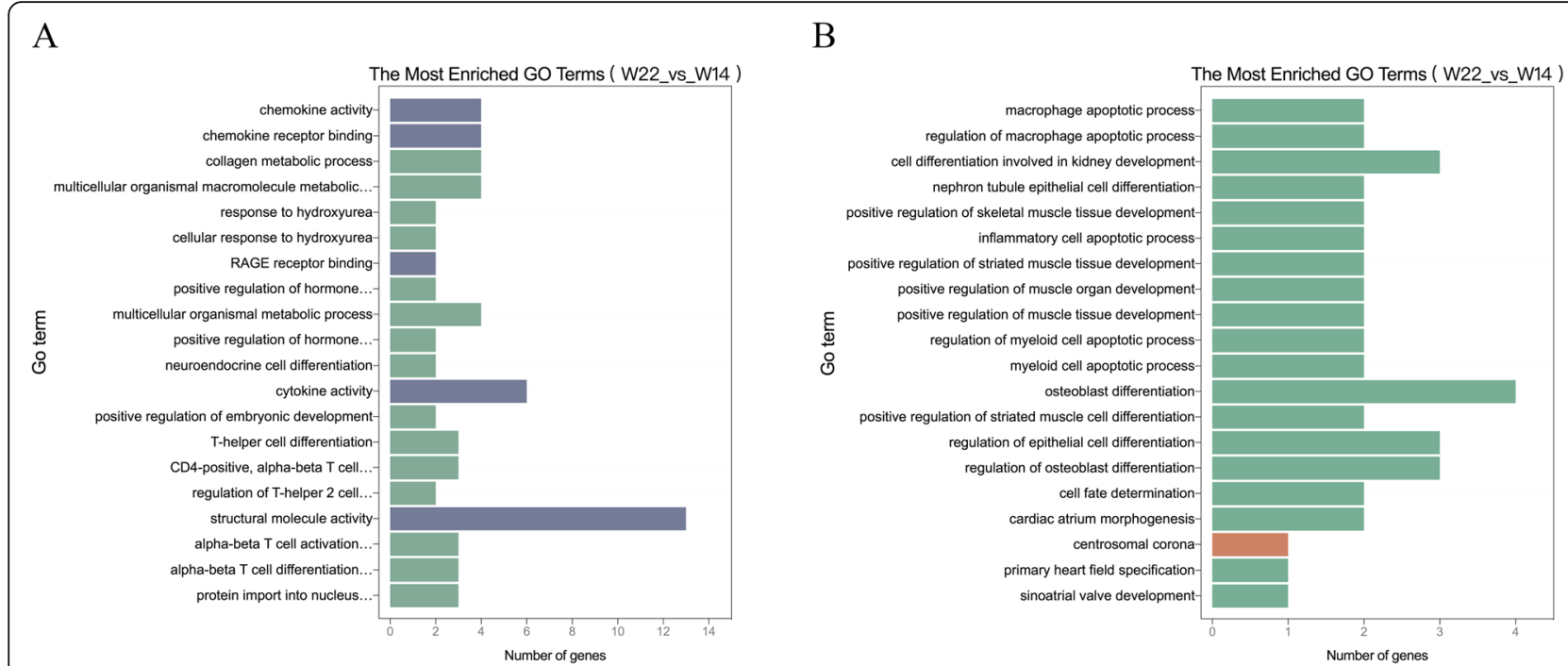

Fig. 4 The enriched GO terms of the DE-IncRNA. a Cis-target genes in the W22 vs. W14 comparison groups. b Trans-target genes in the W22 vs. W14 comparison groups

the W14 vs. W6 comparison group (Fig. S4D). In the W22 vs. W14 comparison group, the two top pathways were arginine and proline metabolism and the MAPK signaling pathway (Fig. 5a). In addition, for the W30 vs. W22 comparison group, there were two top pathway terms, the MAPK signaling pathway and the regulation of actin cytoskeleton pathway (Fig. $5 b)$. We found that the MAPK signaling pathway was one of the most frequently enriched pathways in both the W22 vs. W14 and W30 vs. W22 comparison groups.

\section{Interactions between IncRNAs and mRNAs during breast} muscle development

To explore how lncRNAs interact with their target genes to regulate chicken muscle development and to identify key molecular players in the process, we first predicted the cis- and trans-targets of DE-lncRNAs and then constructed the regulatory networks between DE-IncRNAs and their target genes. A total of 13,460 cis-regulatory interaction relationships were detected between 2309 lncRNAs and 7783 mRNAs (Table S2). In addition, 13, 343 trans-regulatory interaction relationships were
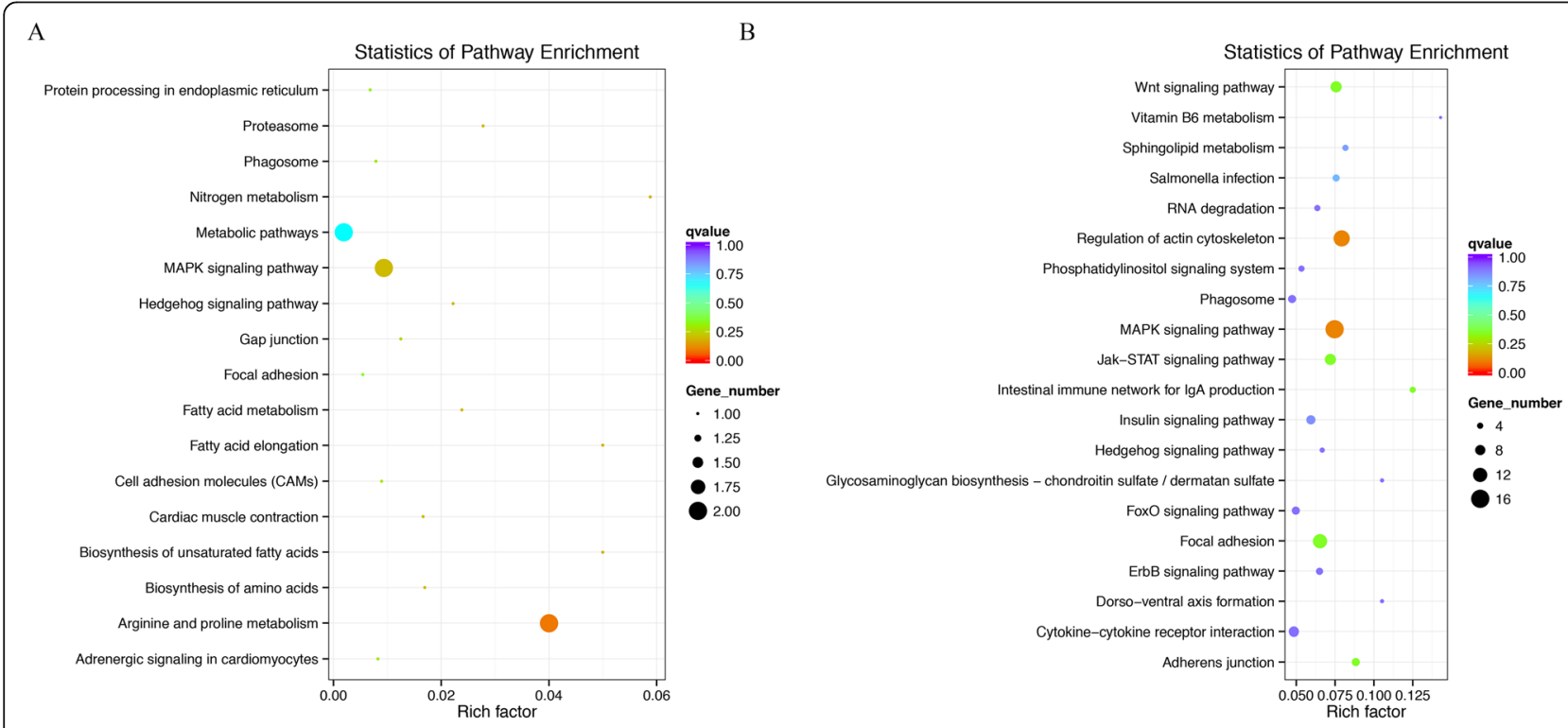

Fig. 5 The enriched KEGG pathways of the DE-IncRNA. a Trans-target genes in the W22 vs. W14 comparison groups. b Trans-target genes in the W30 vs. W22 comparison groups 
detected between 733 lncRNAs and 2190 mRNAs (Table S3). Moreover, we constructed the IncRNA-mRNA networks of cis- and trans-targets for muscle development related to the top $20 \mathrm{GO}$ terms, including positive regulation of embryonic development, positive regulation of skeletal muscle tissue development, positive regulation of striated muscle tissue development, positive regulation of muscle organ development, positive regulation of muscle tissue development and positive regulation of striated muscle cell differentiation. In the networks of cis-target DEGs of DE-lncRNAs of the muscle development-related GO terms, we found a total of 10 interaction relationships between 3 genes and 10 lncRNAs (Fig. 6a). In addition, in the networks of transtarget DEGs of DE-lncRNAs of the muscle development-related GO terms, we found 7 interaction networks between 3 genes and 7 lncRNAs (Fig. 6b). Furthermore, we also generated the IncRNA-mRNA networks of the frequently enriched MAPK signaling pathway, which had a total of 25 interaction relationships between 11 genes and 25 cis-regulating lncRNAs and 27 interaction relationships between 8 genes and 17 trans-regulating lncRNAs (Fig. 6c). Interestingly, we found that the networks containing $M E F 2 C$ and its targeting lncRNAs (ALDBGALT0000008862, ALDBGALT0000008865, LNC_001247) were not only in the muscle development-related GO terms but also in the MAPK signaling pathway.

\section{LncRNA-miRNA interactions}

In our previous study [19], we found 388 known miRNAs and 31 novel miRNAs. To explore the interactions between IncRNAs and miRNAs, we predicted the target relationship between lncRNAs and miRNAs in different comparison groups (Table S4). Twelve common target DE-miRNAs of DE-lncRNAs were found in different comparison groups. It is important that some of them were muscle-specific miRNAs, such as gga-miR-206, gga-miR-1a-3p, and miR-133a-3p. Only the lncRNAs (FPKM $>1$ ) of these miRNA targets are shown in Fig. 7. Then, we predicted the pre-miRNAs with homology to lncRNAs. Unexpectedly, the precursors of four newly identified miRNAs were found to be homologous to IncRNAs, and the precursors were temporarily named gga-miR-N1, gga-miR-N2, gga-miR-N3 and gga-miR-N4 (Fig. 8, Table S5). For example, the pre-miRNA of ggamiR-N1 exactly matches ALDBGALT0000008009 at its position from 309 to 374 , and the pre-miRNA of ggamiR-N2 exactly matches lnc_000010 at its position from 1527 to 1587, and it also matches lnc_000011 from 1101 to 1161 . These lncRNAs may form miRNA precursors through intracellular shearing and then could be processed to generate specific miRNAs that regulate the expression of target genes.

\section{LncRNA-miRNA-mRNA regulatory networks}

To identify potential ceRNA networks in the development of chicken breast muscle, we constructed ceRNA networks of DEGs, differentially expressed miRNAs (DEMs), and DElncRNAs ( $q$-value $<0.05$ and $\log 2|\mathrm{FC}| \geqq 1$ ) by Cytoscape (Fig. S5), and we found some ceRNA networks associated with muscle development-related GO terms (Fig. 9). For example, 445 ceRNA networks were found in the lncRNA-miRNAmRNA network in the W14 vs. W6 comparison group (Fig. S5A). Among these networks, ankyrin repeat domain 1 (ANKRD1) is related to skeletal muscle cell differentiation, and it was involved in 13 ceRNA networks containing two miRNAs (miR-148a-3p and miR-10b-5p) and 12 lncRNAs (LNC_000846, ALDBGALT0000001052, LNC_000453, LN C_001182, ALDBGALT0000006695, ALDBGALT0000002 546, ALDBGALT0000006376, LNC_000255, LNC_000938, ALDBGALT0000006015, LNC_001012, and ALDBGALT0 000000480) (Fig. 9a). In addition, dynein light chain 2 (DYNLL2) is related to the myosin complex GO term, which was involved in 19 ceRNA networks with two miRNAs (ggamiR-148a-3p and gga-miR-130b-3p) and 12 lncRNAs (LNC 000846, ALDBGALT0000001052, LNC_000453, LNC_ 001182, ALDBGALT0000006695, ALDBGALT0000002546, ALDBGALT0000006376, LNC_000255, LNC_001012, LN C_000938, ALDBGALT0000006015, and ALDBGALT000 0003517) (Fig. 9a). In the W22 vs. W14 comparison group, there were 76 ceRNA networks (Fig. S5B). Among them, myosin heavy polypeptide 11 (MYH11) is related to muscle cell differentiation, which was involved in 8 ceRNA networks containing gga-miR-194 and 8 lncRNAs (LNC_000668, LNC_000569, LNC_001009, ALDBGALT0000000938, LN C_001086, LNC_000373, LNC_000920, and LNC_001140) (Fig. 9b). Moreover, there were 450 ceRNA networks in the W30 vs. W22 comparison group (Fig. S5C). The skeletal muscle fiber development-related gene regulators of calcineurin 1 (RCAN1) and ANKRD1 were involved in 8 ceRNA networks containing gga-miR-92-3p and 8 lncRNAs (LNC_ 000920, LNC_000704, ALDBGALT0000001001, ALDBGAL T0000005521, LNC_000618, ALDBGALT0000000349, LNC _000204, and ALDBGALT0000003603) (Fig. 9c).

\section{Protein-protein interaction (PPI) network of DE-IncRNA target genes}

The PPI network was constructed by Cytoscape software using the predicted protein-protein interaction networks from the STRING database (Fig. 10). The PPI network from DE-lncRNA cis-target genes of the W14 vs. W6 comparison group contained 13 protein-protein pairs, such as IGF-I-EGF. Moreover, in the W22 vs. W14 comparison group, there were 6 protein-protein pairs, for example, FZD6-WNT11. Furthermore, the DEGs from the $W 30$ vs. W22 comparison group included 23 proteinprotein pairs, including AR-PPAR. However, no PPI network was found in the DE-lncRNA trans-target genes of 
A

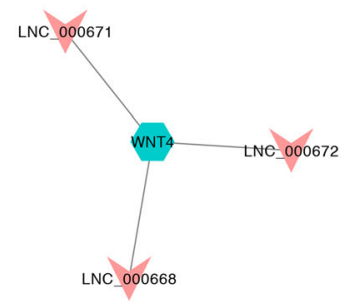

B

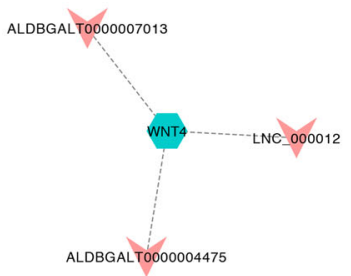

gene
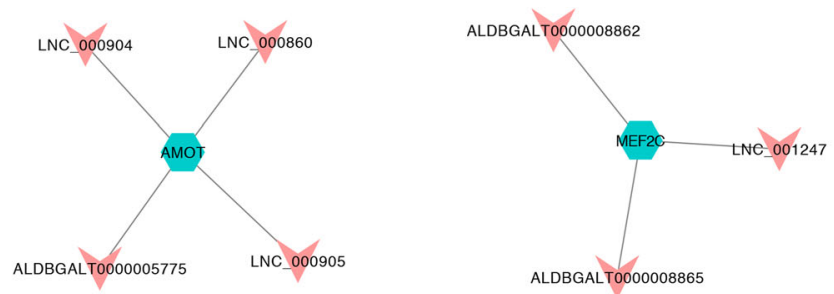

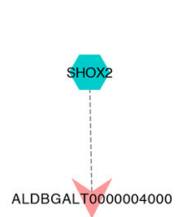

ALDBGALTOD00006376
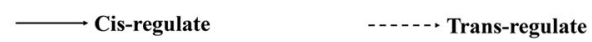

C

MAPK signaling pathway

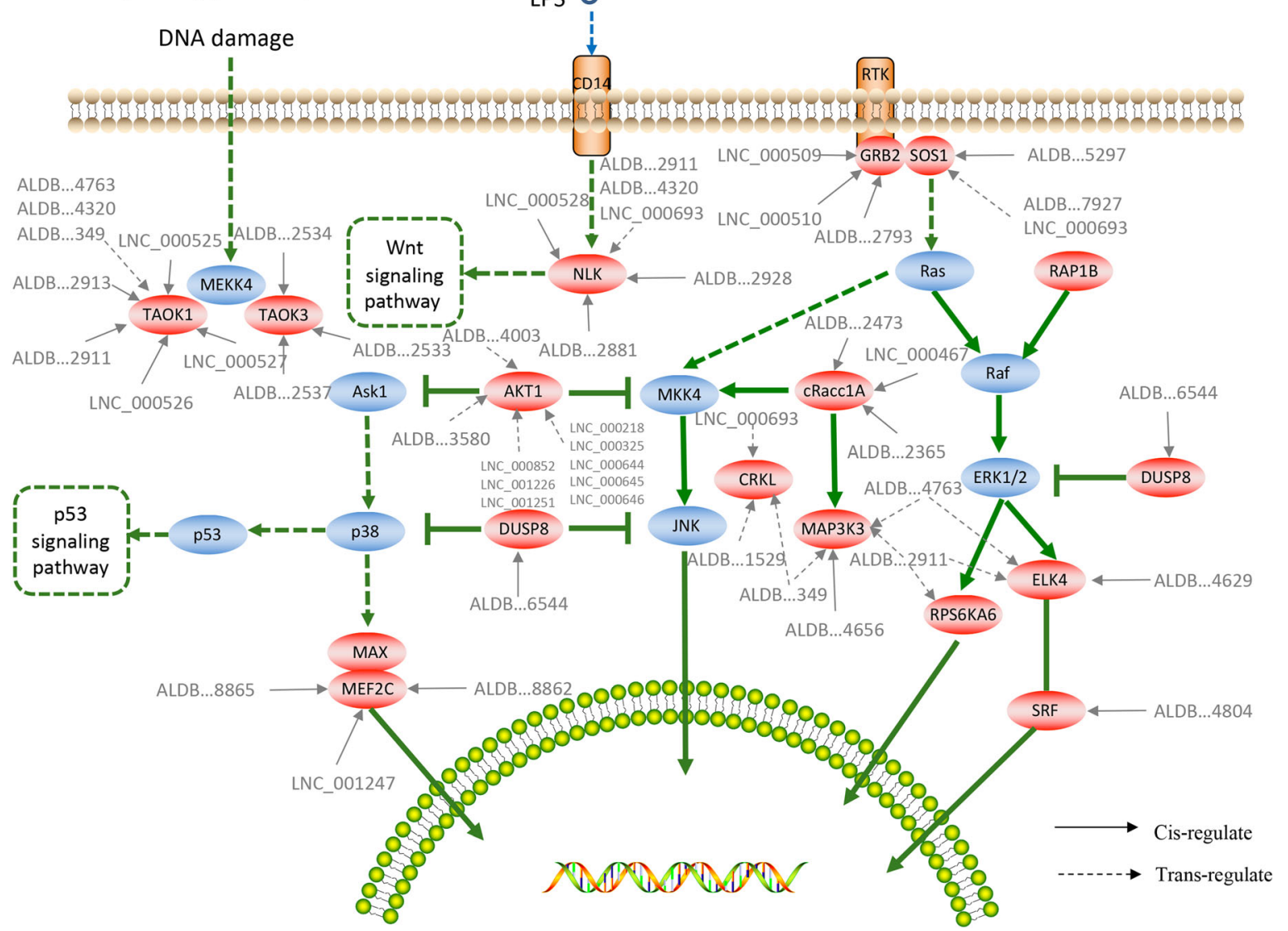

Fig. 6 The cis- and trans-networks of DEGs and DE-IncRNAs of muscle development-related GO terms and signaling pathways. a The cis-target networks associated with enriched GO terms. b The trans-target networks associated with enriched GO terms. c The networks of cis- and trans-target DEGs of DEIncRNAs ( $r>0.98$ ) in the MAPK signaling pathway (The network relations were refer to the KEGG website: https://www.genome.jp/kegg/pathway.html. Only the red nodes represent the DE IncRNA-targeted genes. Solid arrows represent cis-targets, and dashed arrows represent trans-targets) 

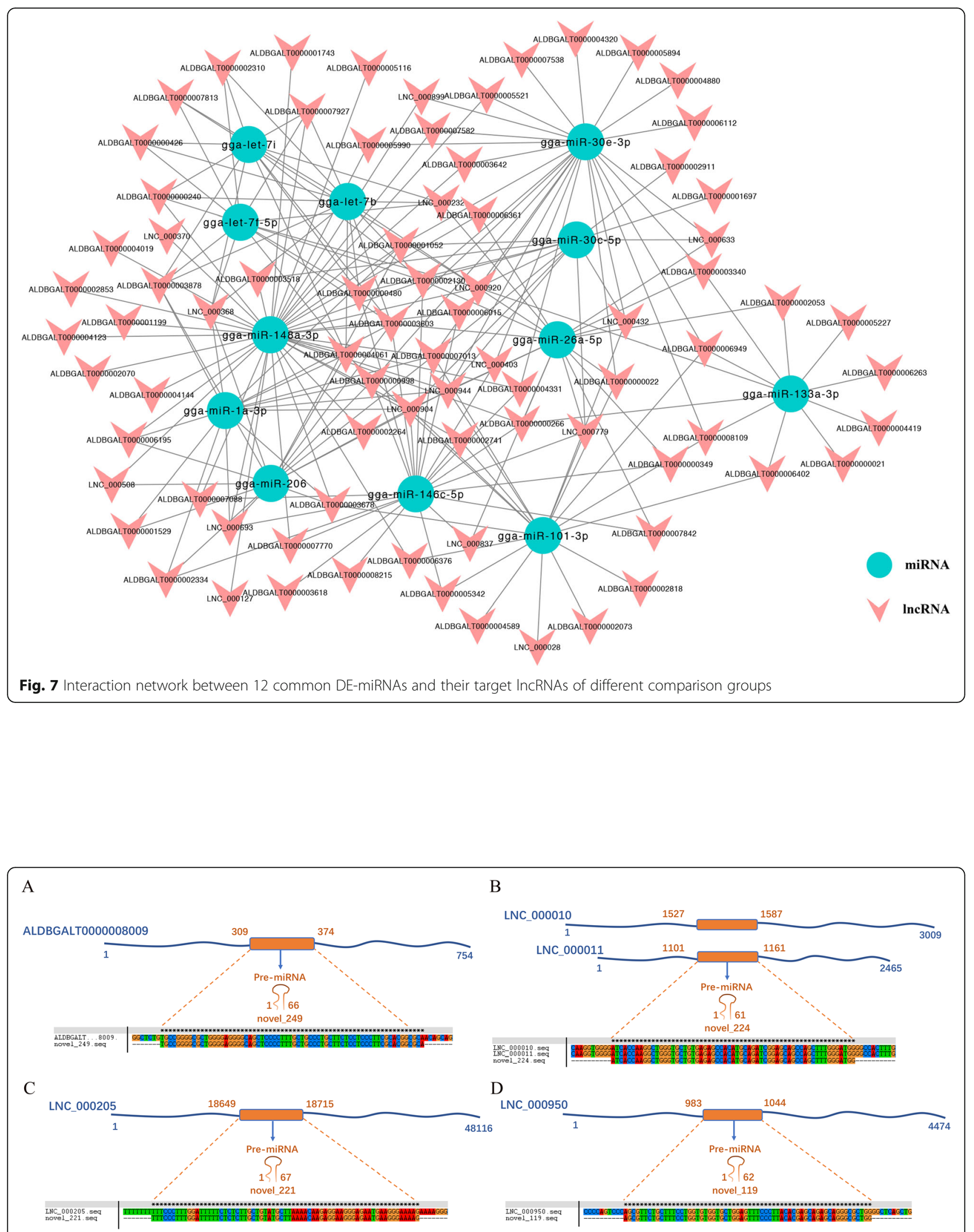

Fig. 8 Homology of IncRNAs with pre-miRNAs. a Homology of ALDBGALT0000008009 with gga-miR-N1. b Homology of Inc_000010 and Inc_000011 with gga-miR-N2. c Homology of Inc_000205 with gga-miR-N3. d Homology of Inc_000950 with gga-miR-N4 

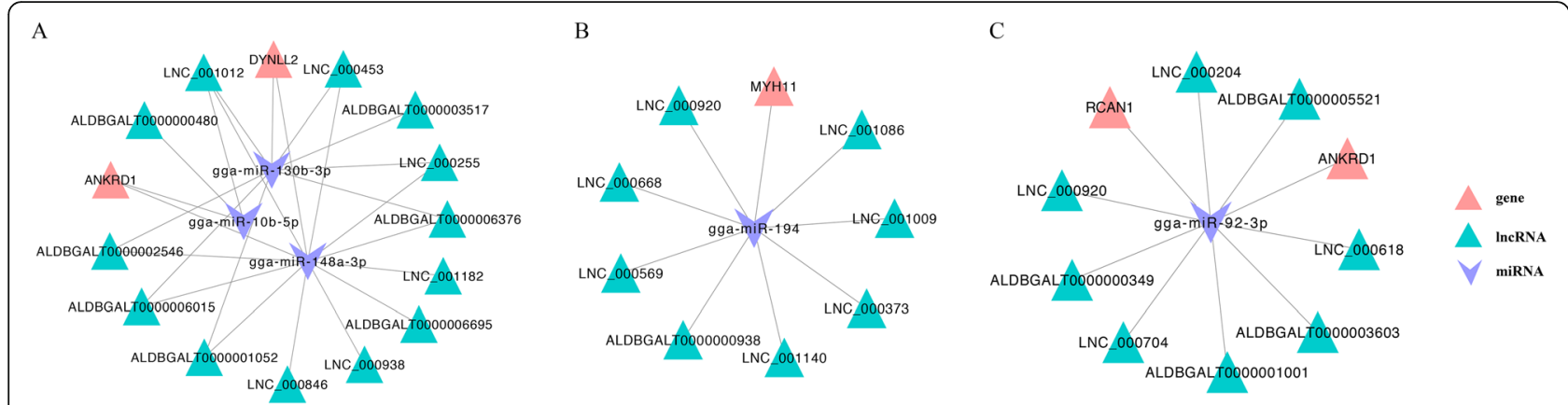

Fig. 9 The IncRNA-miRNA-mRNA ceRNA networks of muscle development-related GO terms. a W14 vs. W6 comparison group; (b) W22 vs. W14 comparison group; (c) W30 vs. W22 comparison group

the W14 vs. W6 and W22 vs. W14 comparison groups. In addition, the DE-lncRNA trans-targets from the W3O vs. W22 comparison group included 36 protein-protein pairs, including $C D K 8-C C N C$.

\section{Discussion}

The growth and development of skeletal muscle is regulated by many factors, such as nutrition, genetics, and the environment. In genetics research, previous studies focused on the role of protein-coding genes in skeletal muscle growth and development, and it was later found that noncoding RNA represents the majority of the transcriptome, with studies showing that only $<2 \%$ of mammalian genomes encode proteins [21]. The existence of a large number of noncoding RNAs must have value and significance. Noncoding RNAs, including miRNAs [22], IncRNAs [23, 24], and circRNAs [25], play different roles in complex biological processes. However, there are few studies on lncRNAs in chicken skeletal muscle development. In our study, we compared the differences in lncRNA expression in chicken breast muscles of chickens at four different ages $(6,14,22$, and 30 weeks) to identify important lncRNAs associated with muscle development.

Our research showed that chromosomal distribution of DE-lncRNAs was mainly concentrated in chromosome 1 , which was similar to previous studies, i.e., there were more lncRNAs distributed in larger chromosomes [26]. The DE-lncRNAs on these chromosomes may exert their effects by regulating the expression of adjacent functional genes. Recent studies have demonstrated that lncRNAs can control the expression of important genes through cis- and trans-regulatory mechanisms [27]. In the present study, functional enrichment analyses of both cis- and trans-target genes of DE-lncRNAs revealed that the muscle development-related enriched GO terms were found only in the W22 vs. W14 comparison group. This is consistent with our previous transcriptomics analysis [19] and indicates that the period from 14 to 22 weeks is an important stage in the development of chicken breast muscle. In addition, in the pathway enrichment analysis of the DE-lncRNA trans-target genes,

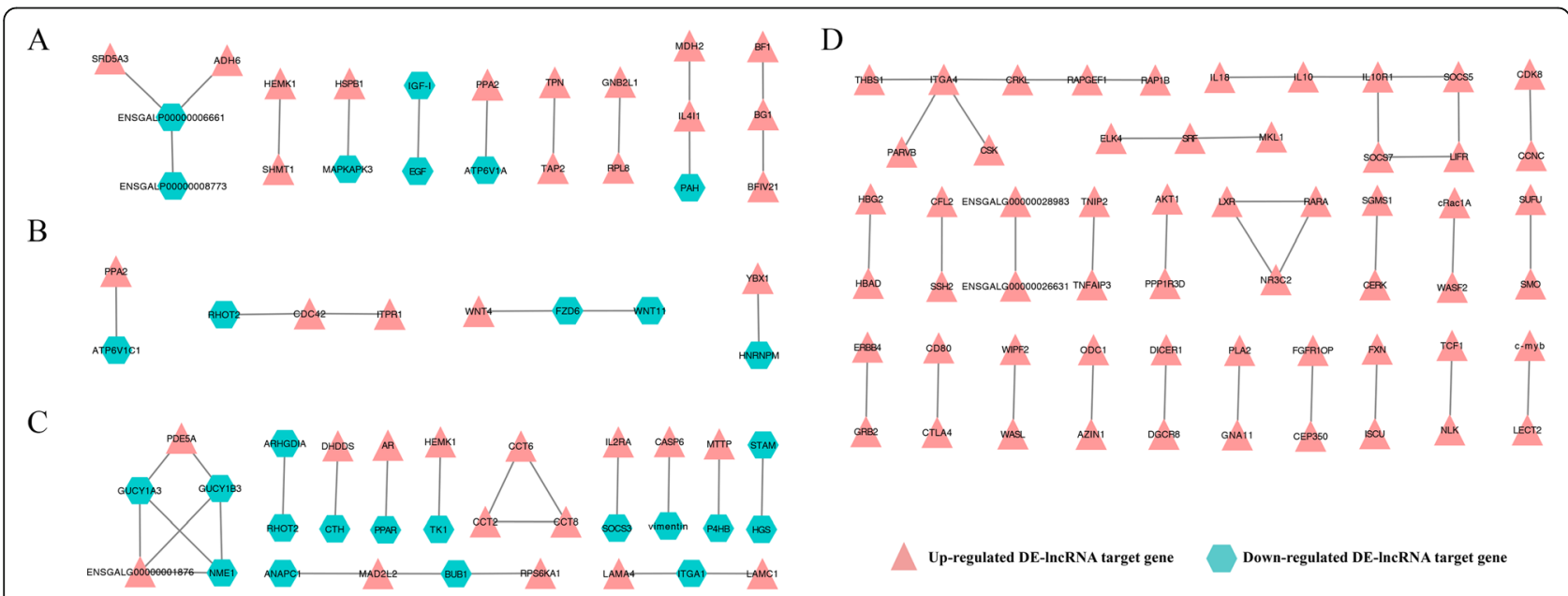

Fig. 10 The PPI network of DE-IncRNA target genes. (a) Cis-target genes of the W14 vs. W6 comparison group; (b) Cis-target genes of the W22 vs. W14 comparison group; (c) Cis-target genes of the W30 vs. W22 comparison group; (D) Trans-target genes of the W30 vs. W22 comparison group 
it was found that there was a common pathway in the W22 vs. W14 and W30 vs. W22 comparison groups, the MAPK signaling pathway. The MAPK family plays crucial roles in complex cellular processes, such as proliferation, differentiation, and development, by regulating the cell cycle and other cell proliferation-associated proteins [28]. We speculate that the DE-lncRNAs in the W22 vs. W14 and W30 vs. W22 comparison groups may play important roles in regulating muscle cell proliferation. Moreover, the function of lncRNAs can be inferred from associated contiguous or co-expressed protein-coding genes [29]. Thus, we constructed interaction networks of DE-lncRNAs and their cis- and trans-target genes, and only some of the genes associated with muscle development-related GO terms and the MAPK signaling pathway and their corresponding lncRNAs are shown in Fig. 6. Interestingly, we found that the networks containing $M E F 2 C$ and its targeting lncRNAs were not only in the muscle development-related GO terms but also in the MAPK signaling pathway. Research has shown that $M E F 2$ factors are involved in muscle differentiation, and $M y o D$ and $M E F 2$ family members interact to activate transcription and myogenesis [30]. Thus, we speculate that these lncRNAs, such as ALDBGALT0000008862, ALDBGALT0000008865, and LNC_001247, are involved in muscle differentiation.

LncRNAs have significant similarities to classic mRNAs during transcription, so miRNAs can not only target mRNAs but also regulate target lncRNAs and reduce their structural and functional stability [31]. It has been speculated that miRNA-192 and miRNA-204 directly suppress lncRNA HOTTIP and interrupt GLS1mediated glutaminolysis in hepatocellular carcinoma [32]. Interestingly, miRNAs can also enhance lncRNA expression through several mechanisms [33]. miR-140 binding sites were identified in NEAT1, and the authors found that mature miR-140 could physically interact with NEAT1 in the nucleus, thereby promoting the expression of NEAT1 [34]. The predicted miRNA-lncRNA networks are shown in Fig. 7 and suggest that miRNAs may play a role in promoting or inhibiting lncRNA expression. Interestingly, several muscle-specific miRNAs, such as gga-miR-206, gga-miR-1a-3p, and miR-133a-3p, have been identified [35]. Therefore, we speculate that the target nncRNAs of these miRNAs may also be involved in the muscle development process. In addition, lncRNAs play many complex roles in gene expression and signal transduction and can also be used as precursors of miRNAs. LncRNAs can produce specific miRNAs through intracellular RNA splicing to affect the posttranscriptional regulation of mRNAs. It has been found that the H19 RNA is a miRNA precursor and generates the exonic microRNA miR-675 [36]. In this study, we found 5 pairs of homologous lncRNAs and pre-miRNAs, including ALDBGAL0000008009 with gga-miR-N1 and lnc_000010 and lnc_000011 with gga-miR-N2. We believe that the miRNAs produced by these lncRNAs play a functional role.

There is increasing evidence that supports the ceRNA hypothesis that lncRNAs regulate target genes by competitively adsorbing miRNAs [37]. For instance, IncRNAUnigene56159 can directly bind to miR-140-5p and act as a ceRNA of miR-140-5p to regulate the expression of its target gene Slug, thereby affecting HCC cell migration and invasion [38]. Furthermore, lncRNA-MEG3 acts as a tumor suppressor and a ceRNA that regulates E-cadherin and forkhead box $\mathrm{O} 1$ (FOXO1) expression by competitively binding to miR-9 [39]. In thyroid cancer, lncRNA-Gas5 regulates phosphate and tension homology deleted on chromosome ten (PTEN) expression through a ceRNA mechanism as a sponge for miR-2223p [40]. To fully identify how lncRNA-associated ceRNA networks affect breast muscle development in different developmental stages, we predicted and successfully constructed a network of ceRNAs associated with DElncRNAs in different comparison groups. For example, in the $W 14$ vs. W6 comparison group, there were ceRNA networks containing the DYNLL2 gene with 12 lncRNAs that targeted 2 miRNAs, gga-miR-148a-3p and gga-miR130b-3p (Fig. 9a). DYNLL2 is involved in a variety of cellular processes and interacts with myosin $5 \mathrm{a}$ (myo5a) to participate in cargo transport [41]. Therefore, we speculate that the above 12 IncRNAs may regulate DYNLL2 by adsorbing miR-148a-3p and gga-miR-130b-3 to regulate muscle development. Furthermore, the ceRNA network analysis showed that ANKRD1 is involved in 13 ceRNA networks containing two miRNAs (miR-148a-3p and miR-10b-5p) and 12 lncRNAs in the W14 vs. W6 comparison group (Fig. 9a). Moreover, ANKRD1 was involved in 8 ceRNA networks containing gga-miR-92-3p with 8 lncRNAs in the W30 vs. W22 comparison group (Fig. 9c). Studies have shown that ANKRD1 can induce gene expression in cultured skeletal muscle cells and trigger signaling via myogenic regulators $(M R F s)$ during myogenesis [42]. Therefore, we think that the above 20 lncRNAs can also play roles in skeletal muscle cells to regulate the ANKRD1 gene by adsorbing miR-148a-3p, miR-10b-5p, and gga-miR-126-5p.

In addition, we also demonstrated the interactions between DE-lncRNA target genes in Gushi chicken breast development. The interaction between IGF-I and epidermal growth factor $(E G F)$ was identified in the W14 vs. W6 comparison group. It has been reported that the expression of the IGF gene is enhanced during muscle hypertrophy and that locally produced IGF-I may play roles in skeletal muscle growth [43]. Heparin-binding epidermal growth factor (HB-EGF) promotes airway smooth muscle (ASM) cell proliferation through the 
MAPK pathway [44]. Thus, we surmise that IGF-I and $E G F$ can interact to play a role in skeletal muscle cell proliferation. Moreover, the PPI networks based on the W22 vs. W14 comparison group included FZD6-WNT11 among others. The WNT/planar cell polarity (PCP) signaling pathway is involved in the development of human cancer. In this signaling pathway, winglesstype MMTV integration site family 11 (WNT11) can transduce PCP signals through receptors such as frizzled homolog 6 (FZD6) [45]. Thus, we speculate that FZD6 and WNT11 can interact to play a role in cell proliferation. In addition, some of the DE-lncRNA trans-targets from the W30 vs. W22 comparison group were included in the CDK8-CCNC PPI network. Research has shown that cyclin-dependent kinase $8(C D K 8)$ and cyclin $\mathrm{C}(\mathrm{CCNC})$ are transcriptional regulators that mediate several oncogenic pathways [46]. Therefore, the interactions between these genes may eventually affect muscle development, and the above results indicate that there are complex intergenic interactions in the development of chicken breast muscle.

\section{Conclusions}

In conclusion, we first described the IncRNA profile of Gushi chicken breast muscle development at 6, 14, 22, and 30 weeks. In the W22 vs. W14 comparison groups, some GO terms related to muscle development were found, further indicating that between 14 and 22 weeks, changes in the expression of some crucial lncRNAs and their target genes affected the growth and development of chicken breast muscles during these important stages. In addition, the MAPK signaling pathway was found to play a vital role in muscle development. $M E F 2 C$ and its target lncRNA, such as ALDBGALT0000008862, ALDBGALT0000008865, and LNC_001247, may be involved in muscle regulation through the MAPK signaling pathway. These results provide insight and valuable resources for further research on the molecular mechanisms of skeletal muscle development after hatching.

\section{Methods}

\section{Animals and sample preparation}

The experimental animals in this study were Gushi chickens from the Animal Center of Henan Agricultural University. A total of 300 one-day-old female Gushi chickens were raised in cages with the same environment, with standard conditions for pure breeding conservation of Gushi chickens. In this study, three healthy chickens were randomly selected at $6,14,22$, and 30 weeks of age. Therefore, twelve chickens were used in this study. Our sample size was sufficient, and the remaining healthy chickens are still used for the pure breeding conservation of Gushi chickens. These chickens had a two-stage feeding protocol, in which $18.5 \%$ crude protein and $12.35 \mathrm{MJ} / \mathrm{kg}$ were prepared in the first stage (younger than 14 weeks) and $15.6 \%$ crude protein and $12.75 \mathrm{MJ} / \mathrm{kg}$ were prepared in the second stage (older than 14 weeks), and the chickens had free access to water. Chickens were anesthetized by intravenous injection of sodium pentobarbital $(40 \mathrm{mg} / \mathrm{kg})$ at a concentration of $0.2 \%$ in the wing vein. Under deep anesthesia, these individuals were euthanized by intravenous $\mathrm{KCL}$ $(1-2 \mathrm{mg} / \mathrm{kg})$. The left breast muscle tissue was then collected, immediately frozen in liquid nitrogen and stored at $-80^{\circ} \mathrm{C}$ until RNA extraction.

\section{Illumina deep sequencing and sequence analysis}

Twelve RNA libraries were constructed using 12 breast muscle samples (W6, W14, W22, and W30; each stage had three individual samples). Total RNA was isolated from breast muscle samples using TRIzol reagent (Invitrogen, Carlsbad, CA, USA) following the manufacturer's instructions. The quantity and quality of RNA were evaluated by NanoDrop 2000 (Thermo Scientific, Wilmington, DE, USA) and by $1 \%$ agarose gel electrophoresis. A total of $3 \mu \mathrm{g}$ RNA per sample was used as input material for the cDNA library. First, the Epicentre Ribo-zero ${ }^{\text {tw }}$ rRNA Removal Kit (Epicentre, Madison, Wisconsin, USA) was used to remove ribosomal RNA. Second, sequencing libraries were generated using rRNA-depleted RNA with the NEBNext ${ }^{\circ}$ Ultra $^{\mathrm{m}}$ Directional RNA Library Prep Kit for Illumina ${ }^{\circ}$ (NEB, Ipswich, MA, USA) following the manufacturer's instructions. The products were then purified using a TruSeq RNASample Prep Kit v2 (New England Biolabs, Ipswich, MA, USA), and library quality was assessed on an Agilent Bioanalyzer 2100 system (Agilent Technologies, CA, USA). Finally, the libraries were sequenced using an Illumina HiSeq 2500 platform, and paired-end reads were generated. The raw data in the fastq format were first processed by in-house scripts. The Illumina sequencing raw reads were obtained by removing adapter sequences, reads with poly$\mathrm{N}$ and low-quality reads, in which the number of bases with a quality value $\mathrm{Q} \leq 20$ was $>50 \%$. All downstream analyses were based on high-quality clean data. Reference genome and gene model annotation files were downloaded from a genome website (ftp://ftp.ensembl. org/pub/release-83/fasta/gallus_gallus/dna/). An index of the reference genome was built using Bowtie v2.2.3, and paired-end clean reads were aligned to the reference genome using TopHat v2.0.12. The Cufflinks v2.1.1 Reference Annotation Based Transcript (RABT) assembly method was used to construct and identify transcripts from the TopHat alignment results.

\section{Identification of IncRNAs}

The combined transcript sets were screened for lncRNAs, and the lncRNA screening process was divided 
into the following five steps: the first step was to select the transcripts with exon number $\geq 2$; the second step was to select the transcripts with transcript length $>200$ bp; the third step was to use Cuffcompare software to filter out the transcripts that overlapped with exon regions annotated in the database, and the lncRNAs in the database that overlapped with the exon regions of splicing transcripts were annotated as lncRNAs in subsequent analyses; the fourth step was to calculate the expression of each transcript by Cuffquant, whereby transcripts with an FPKM (fragments per kilobase of exons per million mapped fragments) $\geq 0.5$ expression level were selected; and the fifth step was to screen the protein-coding potential, in which the four databases CPC, PFAM, PhyloCSF and CNCI were used to remove potential protein-coding transcripts. In this study, the resulting transcripts with no protein-coding potential in the software analyses resulted in the IncRNA dataset, and the transcripts that were predicted to have proteincoding potential by at least one coding potential prediction software were set as TUCPs.

\section{Differential expression analysis}

Cuffdiff (v2.1.1) was used to calculate the FPKM of IncRNAs in each sample, and cuffdiff used a model based on a negative binomial distribution to provide statistical procedures for determining differential expression in digital transcript or gene expression data [20]. Based on Illumina sequencing data, FPKM values were used to assess the expression levels of lncRNAs in the libraries constructed from breast muscle. The FC for each lncRNA between two discretionary stages was calculated according to comparisons between four comparison groups (W6, W14, W22, and W30). Differential expression analysis among the four groups was performed using the DESeq $\mathrm{R}$ package (1.8.3), and lncRNAs and genes found by DESeq with a $q$-value $<0.05$ and $|\mathrm{FC}| \geq 1.7$ were considered to be differentially expressed.

\section{Cis- and trans-targeting analyses}

Differentially expressed lncRNAs were selected for cisand trans-target gene predictions and were integrated with differentially expressed gene data to improve the veracity of target prediction. In the present study, DEGs located $\sim 100 \mathrm{~kb}$ upstream and downstream of DElncRNAs were classified as cis-acting target genes. In addition, we predicted the regulation in trans between lncRNAs and genes by the Pearson correlation coefficient, $r>0.95$. The relationship between lncRNAs and their target genes was demonstrated by Cytoscape 3.4 (http://www.cytoscape. org/).

All potential target genes for DE-lncRNAs in each comparison group were used in the bioinformatics analysis. GO and KEGG pathway analyses were conducted using the DAVID database [47] to visualize data. Only GO terms and KEGG pathways with corrected $p$-values $(t$-test $)<0.05$ were considered significantly enriched.

\section{Association analysis and interaction network construction} The data including mRNAs [3] and miRNAs [19] obtained from our previous study were used for integrative analysis with the lncRNA data. The transcriptome and small RNA library were constructed using the same tissue RNA Gushi chicken breast muscle samples from chickens at the four developmental stages in our previous research. Additionally, a $q$-value $<0.05$ was set as the threshold for significant DEMs by default. DEMs, DEGs, and DE-lncRNAs were used to construct lncRNA-miRNA-mRNA interaction networks by Cytoscape 3.4. PPI analysis of DEGs targeted by DE-lncRNAs was based on the STRING database (https://string-db. org, Organism: Gallus gallus). The network of interactions between these DEGs was generated in the STRING database and imported into Cytoscape software for visualization; scores $>700$ were selected as significant interactions to display.

\section{Quantitative real-time PCR (qRT-PCR) analysis}

The qRT-PCR analysis of lncRNAs was performed using the PrimeScript ${ }^{\mathrm{ma}} \mathrm{RT}$ reagent kit (TaKaRa, Dalian, China) according to the manufacturer's instructions. qRT-PCR was performed with a LightCycler 96 instrument qRTPCR system (Roche, Basel, Switzerland) with the PrimeScript $^{\text {tm }}$ RT Reagent Kit with gDNA Eraser (Takara, Kyoto, Japan). The amplification program consisted of $95^{\circ} \mathrm{C}$ for $3 \mathrm{~min} ; 40$ cycles of $95^{\circ} \mathrm{C}$ for $10 \mathrm{~s}$; and annealing at $60^{\circ} \mathrm{C}$ for $30 \mathrm{~s}, 72{ }^{\circ} \mathrm{C}$ for $30 \mathrm{~s}$, and $72^{\circ} \mathrm{C}$ for $10 \mathrm{~min}$. The relative expression levels were analyzed with the $2^{-\triangle \Delta \mathrm{Ct}}$ method. The qRT-PCR primer sequences are listed in Table S6.

\section{Statistical analysis}

The qRT-PCR quantitative expression data and graphs were generated in GraphPad Prism (version 5.0) software (San Diego, CA, USA). Statistical significance of the qRT-PCR quantitative expression data was tested by performing two-tailed unpaired t-tests [48]. Data are presented as the means containing three replicates.

\section{Supplementary Information}

The online version contains supplementary material available at https://doi. org/10.1186/s12864-020-07356-6.

Additional file 1: Fig. S1. Heatmap showing DE-IncRNAs from different stages.

Additional file 2: Fig. S2. The chromosome distribution of DE-IncRNAs from different stages. 
Additional file 3: Fig. S3. The enriched GO terms of the DE-IncRNA. (AB) Cis-target genes in the W14 vs. W6 and W30 vs. W22 comparison groups. (C-D) Trans-target genes in the W14 vs. W6 and W30 vs. W22 comparison groups.

Additional file 4: Fig. S4. The enriched KEGG pathways of the DEIncRNA. (A-C) Cis-target genes in the W14 vs. W6, W22 vs. W14, and W30 vs. W22 comparison groups. (D) Trans-target genes in the W14 vs. W6 comparison groups.

Additional file 5: Fig. S5. The IncRNA-miRNA-mRNA ceRNA networks. (A) W14 vs. W6 comparison group; (B) W22 vs. W14 comparison group; (C) W30 vs. W22 comparison group.

Additional file 6; Table S1. Summary of draft reads of 12 cDNA libraries, determined by RNA sequencing. Abbreviations: W6_1, sample 1 of 6 weeks; W6_2, sample 2 of 6 weeks; W6_3, sample 3 of 6 weeks; W14_1, sample 1 of 14 weeks; W14_2, sample 2 of 14 weeks; W14_3, sample 3 of 14 weeks; W22_1, sample 1 of 22 weeks; W22_2, sample 2 of 22 weeks; W22_3, sample 3 of 22 weeks; W30_1, sample 1 of 30 weeks; W30_2, sample 2 of 30 weeks; W30_3, sample 3 of 30 weeks.

Additional file 7: Table S2. Cis-regulatory interactions between DEIncRNAs and DE-mRNAs.

Additional file 8: Table S3. Trans-regulatory interactions between DEIncRNAs and DE-mRNAs.

Additional file 9: Table S4. The interaction between DE-IncRNAs and DE-miRNAs.

Additional file 10: Table S5. Details on the novel miRNAs identified in this study. Abbreviations: W6, W14, W22, and W30 represent small RNA libraries obtained using samples from chickens aged 6, 14, 22, and 30 weeks, respectively.

Additional file 11: Table S6. aRT-PCR primers. Abbreviation: AT refers to the annealing temperature; $F$ and $R$ refer to the forward and reverse primers, respectively.

\section{Abbreviations}

GHSR: Growth hormone secretagogue receptor; IGFs: Insulin-like growth factors; TGF $\beta 2$ : Transforming growth factor beta 2; MEF2B: Myocyte enhancer factor 2B; IncRNAs: Long noncoding RNAs; MyoD: Myogenic differentiation antigen; IRS1: Insulin receptor substrate 1; TUCPs: Transcripts of uncertain coding potential; lincRNA: Long intergenic noncoding RNAs; DEIncRNAs: Differentially expressed IncRNAs; W6: 6 Weeks; DEG: Differentially expressed gene; GO: Gene ontology; KEGG: Kyoto encyclopedia of genes and genomes; DEMs: Differentially expressed miRNAs; ANKRD1: Ankyrin repeat domain 1; DYNLL2: Dynein light chain 2; MYH11: Myosin heavy polypeptide 11: RCAN1: Regulator of calcineurin 1; FOXO1: Forkhead box O1; PTEN: Phosphate and tension homology deleted on chromosome ten; MRFs: Myogenic regulators; EGF: Epidermal growth factor; HB-EGF: Heparinbinding epidermal growth factor; ASM: Airway smooth muscle; PCP: Planar cell polarity; WNT11: Winglesstype MMTV integration site family 11; FZD6: Frizzled homolog 6; CDK8: Cyclin-dependent kinase 8; CCNC: Cyclin C

\section{Acknowledgments}

Not applicable.

\section{Authors' contributions}

YL performed the data analysis and wrote the main manuscript; WJ and BZ helped to revise the manuscript; $Y C, W J$ and BZ performed the experiments and analyzed the data; GL reviewed and edited the manuscript; $Y Z$ and $Y G$ performed the statistical analysis; GS and RH helped draft the manuscript; ZL and $\mathrm{HL}$ analyzed the partial sequencing data; $Y T$ and $X \mathrm{~L}$ co-designed the research; XK coordinated the research; and GL and XK provided the materials. The author(s) read and approved the final manuscript.

\section{Funding}

This work was supported by grants from the National Natural Science Foundation of China (31572356), the Key Scientific Research Projects of Henan Colleges and Universities (15A230003), the Program for Innovation Research Team of Ministry of Education (IRT16R23) and NSFC-Henan joint grant (U1804107) and Scientific Studio of Zhongyuan Scholars (30601985). The funding bodies had no role in the study design or in any aspect of the collection, analysis and interpretation of the data or in the writing of the manuscript.

\section{Availability of data and materials}

All raw sequences have been deposited in the NCBI database Sequence Read Archive with the accession numbers PRJNA516810 (mRNA and IncRNA) and PRJNA516961 (miRNA).

\section{Ethics approval and consent to participate}

The experiments and animal care were performed according to the Regulations for the Administration of Affairs Concerning Experimental Animals (Ministry of Science and Technology, China, 2004). The protocols were approved by the Institutional Animal Care and Use Committee of Henan Agricultural University, China. In this study, written informed consent was obtained from Henan Agricultural University to use these animals.

\section{Consent for publication}

Not applicable.

\section{Competing interests}

The authors declare that they have no competing interests.

\section{Author details}

${ }^{1}$ College of Animal Science and Veterinary Medicine, Henan Agricultural University, Zhengzhou 450002, China. ${ }^{2}$ Henan Innovative Engineering Research Center of Poultry Germplasm Resource, Henan Agricultural University, Zhengzhou 450002, China.

Received: 5 February 2020 Accepted: 27 December 2020

Published online: 09 January 2021

\section{References}

1. Guller I, Russell AP. MicroRNAs in skeletal muscle: their role and regulation in development, disease and function. J Physiol. 2010;588(Pt 21):4075-87.

2. Jiang C, Shi P, Li S, Dong R, Tian J, Wei J, Luo S. Gene expression profiling of skeletal muscle of nursing piglets. Int J Biol Sci. 2010;6(7):627-38.

3. Li Y, Chen Y, Jin W, Fu S, Li D, Zhang Y, Sun G, Jiang R, Han R, Li Z, et al. Analyses of MicroRNA and mRNA expression profiles reveal the crucial interaction networks and pathways for regulation of chicken breast muscle development. Front Genet. 2019;10:197.

4. Luo W, Nie Q, Zhang X. MicroRNAs involved in skeletal muscle differentiation. J Genet Genomics. 2013;40(3):107-16.

5. Jin S, Chen S, Li H, Lu Y, Xu G, Yang N. Associations of polymorphisms in GHRL, GHSR, and IGF1R genes with feed efficiency in chickens. Mol Biol Rep. 2014:41(6):3973-9.

6. Mohammed RH, Anderton H, Brameld JM, Sweetman D. Effects of insulin like growth factors on early embryonic chick limb myogenesis. PLoS One. 2017;12(10):e0185775.

7. Darzi Niarami M, Masoudi AA, Vaez Torshizi R. Association of single nucleotide polymorphism of GHSR and TGFB2 genes with growth and body composition traits in sire and dam lines of a broiler chicken. Anim Biotechnol. 2014:25(1):13-22.

8. Chen R, Zhuang S, Chen YP, Cheng YF, Wen C, Zhou YM. Betaine improves the growth performance and muscle growth of partridge shank broiler chickens via altering myogenic gene expression and insulin-like growth factor-1 signaling pathway. Poult Sci. 2018;97(12):4297-305.

9. Li Z, Ouyang H, Zheng M, Cai B, Han P, Abdalla BA, Nie Q, Zhang X. Integrated analysis of Long non-coding RNAs (LnCRNAs) and mRNA expression profiles reveals the potential role of LncRNAs in skeletal muscle development of the chicken. Front Physiol. 2016;7:687.

10. Morris KV, Mattick JS. The rise of regulatory RNA. Nat Rev Genet. 2014;15(6): 423-37.

11. Zhou R, Wang $Y X$, Long KR, Jiang AA, Jin L. Regulatory mechanism for IncRNAs in skeletal muscle development and progress on its research in domestic animals. Yi Chuan. 2018:40(4):292-304.

12. Ip JY, Nakagawa S. Long non-coding RNAs in nuclear bodies. Develop Growth Differ. 2012:54(1):44-54.

13. Qin CY, Cai H, Qing HR, Li L, Zhang HP. Recent advances on the role of long non-coding RNA H19 in regulating mammalian muscle growth and development. Yi Chuan. 2017;39(12):1150-7. 
14. Mueller AC, Cichewicz MA, Dey BK, Layer R, Reon BJ, Gagan JR, Dutta A. MUNC, a long noncoding RNA that facilitates the function of MyoD in skeletal myogenesis. Mol Cell Biol. 2015;35(3):498-513.

15. Sui Y, Han Y, Zhao X, Li D, Li G. Long non-coding RNA Irm enhances myogenic differentiation by interacting with MEF2D. Cell Death Dis. 2019; 10(3):181.

16. Cai B, Li Z, Ma M, Wang Z, Han P, Abdalla BA, Nie Q, Zhang X. LncRNA-Six encodes a micropeptide to activate Six 1 in Cis and is involved in cell proliferation and muscle growth. Front Physiol. 2017:8:230.

17. Gabory A, Ripoche MA, Le Digarcher A, Watrin F, Ziyyat A, Forne T, Jammes $H$, Ainscough JFX, Surani MA, Journot L, et al. H19 acts as a trans regulator of the imprinted gene network controlling growth in mice. Development. 2009;136(20):3413-21.

18. Li Z, Cai B, Abdalla BA, Zhu X, Zheng M, Han P, Nie Q, Zhang X. LncIRS1 controls muscle atrophy via sponging miR-15 family to activate IGF1-PI3K AKT pathway. J Cachexia Sarcopenia Muscle. 2019;10(2):391-410.

19. Fu S, Zhao Y, Li Y, Li G, Chen Y, Li Z, Sun G, Li H, Kang X, Yan F. Characterization of miRNA transcriptome profiles related to breast muscle development and intramuscular fat deposition in chickens. J Cell Biochem. 2018;119(8):7063-79

20. Trapnell C, Williams BA, Pertea G, Mortazavi A, Kwan G, van Baren MJ, Salzberg SL, Wold BJ, Pachter L. Transcript assembly and quantification by RNA-Seq reveals unannotated transcripts and isoform switching during cell differentiation. Nat Biotechnol. 2010;28(5):511-5.

21. Wilusz JE, Sunwoo H, Spector DL. Long noncoding RNAs: functional surprises from the RNA world. Genes Dev. 2009;23(13):1494-504.

22. Meng F, Henson R, Wehbe-Janek H, Ghoshal K, Jacob ST, Patel T. MicroRNA21 regulates expression of the PTEN tumor suppressor gene in human hepatocellular cancer. Gastroenterology. 2007;133(2):647-58.

23. Liu Q, Huang JG, Zhou NJ, Zhang ZQ, Zhang AL, Lu ZH, Wu FT, Mo YY. LncRNA loc285194 is a p53-regulated tumor suppressor. Nucleic Acids Res. 2013:41(9):4976-87.

24. Li WY, Jing ZZ, Cheng YY, Wang XN, Li DH, Han RL, Li WT, Li GX, Sun GR, Tian YD, et al. Analysis of four complete linkage sequence variants within a novel IncRNA located in a growth QTL on chromosome 1 related to growth traits in chickens. J Anim Sci. 2020;98(5):skaa122.

25. Lukiw WJ. Circular RNA (circRNA) in Alzheimer's disease (AD). Front Genet. 2013;4:307.

26. Billerey C, Boussaha M, Esquerre D, Rebours E, Djari A, Meersseman C, Klopp C, Gautheret D, Rocha D. Identification of large intergenic non-coding RNAs in bovine muscle using next-generation transcriptomic sequencing. BMC Genomics. 2014;15(1):499.

27. Han L, Zhang KL, Shi ZD, Zhang JX, Zhu JL, Zhu SJ, Zhang AL, Jia ZF, Wang GX, Yu SZ, et al. LncRNA profile of glioblastoma reveals the potential role of IncRNAs in contributing to glioblastoma pathogenesis. Int J Oncol. 2012; 40(6):2004-12

28. Zhang W, Liu HT. MAPK signal pathways in the regulation of cell proliferation in mammalian cells. Cell Res. 2002;12(1):9-18.

29. Li T, Zhang G, Wu P, Duan L, Li G, Liu Q, Wang J. Dissection of myogenic differentiation signatures in chickens by RNA-seq analysis. Genes. 2018;9(1): 34.

30. Dodou E, Xu SM. Black BL: mef2c is activated directly by myogenic basic helix-loop-helix proteins during skeletal muscle development in vivo. Mech Develop. 2003;120(9):1021-32.

31. Ballantyne MD, McDonald RA. Baker AH: IncRNA/MicroRNA interactions in the vasculature. Clin Pharmacol Ther. 2016;99(5):494-501.

32. Staff PG. Correction: fMiRNA-192 and miRNA-204 directly suppress IncRNA HOTTIP and interrupt GLS1-mediated Glutaminolysis in hepatocellular carcinoma. PLoS Genet. 2016;12(1):e1005825.

33. Liang HW, Zhang JF, Zen K, Zhang CY, Chen X. Nuclear microRNAs and their unconventional role in regulating non-coding RNAs. Protein Cell. 2013; 4(5):325-30.

34. Gernapudi R, Wolfson B, Zhang Y, Yao Y, Yang P, Asahara H, Zhou Q. MicroRNA 140 promotes expression of Long noncoding RNA NEAT1 in Adipogenesis. Mol Cell Biol. 2016;36(1):30-8.

35. Kim HK, Lee YS, Sivaprasad U, Malhotra A, Dutta A. Muscle-specific microRNA miR-206 promotes muscle differentiation. J Cell Biol. 2006;174(5): 677-87.

36. Cai $X Z$, Cullen BR. The imprinted $H 19$ noncoding RNA is a primary microRNA precursor. Rna. 2007;13(3):313-6.
37. Ergun S, Oztuzcu S. Oncocers: ceRNA-mediated cross-talk by sponging miRNAs in oncogenic pathways. Tumour Biol. 2015;36(5):3129-36.

38. Lv J, Fan HX, Zhao XP, Lv P, Fan JY, Zhang Y, Liu M, Tang H. Long noncoding RNA Unigene56159 promotes epithelial-mesenchymal transition by acting as a ceRNA of miR-140-5p in hepatocellular carcinoma cells. Cancer Lett. 2016;382(2):166-75.

39. Dong ZM, Zhang A, Liu SN, Lu F, Guo YL, Zhang GQ, Xu FL, Shi YB, Shen SP, Liang J, et al. Aberrant methylation-mediated silencing of IncRNA MEG3 functions as a ceRNA in esophageal Cancer. Mol Cancer Res. 2017;15(7): 800-10.

40. Zhang XF, Ye Y, Zhao SJ. LncRNA Gas5 acts as a ceRNA to regulate PTEN expression by sponging miR-222-3p in papillary thyroid carcinoma. Oncotarget. 2018:9(3):3519-30.

41. Rapali P, Szenes A, Radnai L, Bakos A, Pal G, Nyitray L. DYNLL/LC8: a light chain subunit of the dynein motor complex and beyond. FEBS J. 2011; 278(17):2980-96.

42. Kojic S, Nestorovic A, Rakicevic L, Belgrano A, Stankovic M, Divac A, Faulkner G. A novel role for cardiac ankyrin repeat protein Ankrd1/CARP as a coactivator of the p53 tumor suppressor protein. Arch Biochem Biophys. 2010; 502(1):60-7.

43. DeVol DL, Rotwein P, Sadow JL, Novakofski J, Bechtel PJ. Activation of insulin-like growth factor gene expression during work-induced skeletal muscle growth. Am J Phys. 1990;259(1 Pt 1):E89-95.

44. Wang Q, Li HQ, Yao Y, Lu GH, Wang YH, Xia DJ, Zhou JY. HB-EGF-promoted airway smooth muscle cells and their progenitor migration contribute to airway smooth muscle remodeling in asthmatic mouse. J Immunol. 2016; 196(5):2361-7.

45. Katoh M. WNT/PCP signaling pathway and human cancer (review). Oncol Rep. 2005;14(6):1583-8

46. Broude EV, Gyorffy B, Chumanevich AA, Chen MQ, McDermott MSJ, Shtutman M, Catroppo JF, Roninson IB. Expression of CDK8 and CDK8interacting genes as potential biomarkers in breast Cancer. Curr Cancer Drug Tar. 2015;15(8):739-49.

47. Huang DW, Sherman BT, Lempicki RA. Systematic and integrative analysis of large gene lists using DAVID bioinformatics resources. Nat Protoc. 2009;4(1): 44-57.

48. Campbell M. Two-tailed tests and a statistical significance at 0.05: what do they mean? Midwifery. 2004;20(2):142-3.

\section{Publisher's Note}

Springer Nature remains neutral with regard to jurisdictional claims in published maps and institutional affiliations.
Ready to submit your research? Choose BMC and benefit from:

- fast, convenient online submission

- thorough peer review by experienced researchers in your field

- rapid publication on acceptance

- support for research data, including large and complex data types

- gold Open Access which fosters wider collaboration and increased citations

- maximum visibility for your research: over $100 \mathrm{M}$ website views per year

At $\mathrm{BMC}$, research is always in progress.

Learn more biomedcentral.com/submissions 